\title{
Biosecurity beyond borders
}

\author{
Increasingly globalized food systems increase risk for the spread of infectious disease in animals and zoonotic \\ transmission to humans. Greater international harmonization for measures and governance in the area of \\ biosecurity for infectious disease is needed.
}

\begin{abstract}
nfectious laryngotracheitis (ILT) is a poultry disease that reduces egg production and can result in up to $70 \%$ bird mortality. ILT vaccine development has been an ongoing effort since the 1930s, yet the disease remains prevalent in more than 100 countries (Gowthaman, V. et al. Vet. Q. 40, 140-161; 2020). In 2018 more than 116,000 commercial poultry were affected by an ILT outbreak in Namibia; in a matter of weeks, outbreaks occurred across 3 locations $800 \mathrm{~km}$ apart. More recently, outbreaks of ILT in commercial and backyard poultry flocks in Northern Ireland affected more than 250,000 birds across 16 locations, prompting calls from the Department of Agriculture, Environment and Rural Affairs (DAERA) to all poultry
\end{abstract} keepers to increase biosecurity measures. Strict ILT vaccine protocols must be followed, as live, attenuated vaccines against the virus can revert to virulence and cause ILT outbreaks. The cause of the outbreak in Northern Ireland has not been established yet, but it is likely that the cases in Namibia arose from shortages of the correct vaccine and incomplete vaccination coverage.

Biosecurity measures for controlling animal infectious disease consist of strategic and integrated approaches for analysis and management of risk to human, animal, plant and environmental health. ILT is a notifiable disease and DAERA have provided a biosecurity checklist for bird owners to assess risk on their facilities, highlighting that poultry litter must be securely stored on their premises for as long as possible to allow the virus to reduce over time. Other measures follow avian influenza biosecurity protocols: limiting movements of animals, people and equipment in and out of facilities; following strict disinfection protocols using approved disinfectants; keeping domesticated birds enclosed, separate from wild birds and vermin; and keeping accurate records of all activities on-site, including any changes in poultry health or behaviour. In food systems, the first line of defence against animal disease exists behind the farmgate.
Beyond the farmgate, policy, regulatory frameworks and education are essential for ensuring that biosecurity measures are clearly outlined and implemented. But whilst biosecurity consists of routine animal husbandry tasks on farms, policy and regulation is linked to a wide range of political affairs - national security, budgets, current political priorities, public interest, loopholes and lack of ownership can contribute to a situation where biosecurity measures are not sufficiently supported at governmental level. The World Organisation for Animal Health (OIE), the FAO and the WHO are responsible for coordinating many biosecurity measures at a global level - yet worldwide coordination of livestock, aquaculture and fishery biosecurity remains a substantial challenge. Initiatives such as CABI's Invasive Species Compendium form important hubs of information, providing science-based guidance on animal diseases in an accessible manner - informing policy makers and the public about how animal diseases at farm level not only impact farm productivity, but with zoonotic transmission could significantly affect human health. The recent ILT outbreak demonstrates that vaccines alone are not the pathway to eradication; biosecurity is essential - and that requires harmonized action including all players in the system.

The eradication of rinderpest is a notable example of how international cooperation and solidarity can reform animal welfare, human health and food production. Considered the deadliest cattle disease in history, rinderpest spread rapidly from Central Asia to Europe through international trade, causing cattle to experience fever, mouth lesions, nose and eye discharge, diarrhoea, dehydration and, eventually, animal death. Vaccination efforts started as early as 1744 , but strict movement controls and mass vaccination drives in the 1960s formed a substantial part of the efforts to stamp out the disease. The reappearance of rinderpest in Africa in the 1980s prompted the formation of the Global Rinderpest Eradication Programme, a collaboration between the OIE, UN, FAO and the UN International Atomic Energy Agency - in 2011 the disease was declared eradicated worldwide. Ongoing measures through the Rinderpest Vigilance campaign ensures that everyone involved in animal health is fully aware of the challenges linked with a resurgence of the disease and of the vital role that everyone can play to preserve the legacy of eradication. International cooperation and accountability for rinderpest education, surveillance - and regulated scientific research - is at the core of this programme.

In increasingly globalized food systems, transboundary animal diseases require integrated and internationally harmonized approaches to uphold biosecurity actions - and a framework of accountability must be built into food systems. Those expected to uphold the values and implement the practices of biosecurity - especially those with limited means to do so - must be fully supported. Realistic policy and guidelines that function at intensive, commercial farms as well as for smallholders can incentivize compliance, which is especially important in regions that rely on smallholder farmers for food security. Veterinary science and animal welfare research is a crucial component of this approach - informing government advisory services, food safety inspectors, and border and customs officers about best practices for mitigating ongoing and emerging food animal biosecurity threats. With a growing interest in sustainable food systems, consumer demand can drive support for better biosecurity practices - which in turn can improve animal welfare, reduce the risk of antimicrobial resistance development and promote ethical supply chains. Sufficient dietary protein provisioning, mitigating the spread of animal infectious disease and reducing the risk of zoonotic pathogen transmission requires joined-up thinking and international harmonization.

Published online: 21 July 2021

https://doi.org/10.1038/s43016-021-00332-7 